\title{
Wei-Norman equations for a unitary evolution
}

\author{
Szymon Charzyński ${ }^{1}$ and Marek Kuśs \\ ${ }^{1}$ Faculty of Mathematics and Natural Sciences, \\ Cardinal Stefan Wyszyński University, \\ ul. Wóycickiego 1/3, 01-938 Warszawa, Poland \\ ${ }^{2}$ Center for Theoretical Physics, Polish Academy of Sciences, \\ Aleja Lotników 32/46, 02-668 Warszawa, Poland
}

February 12, 2013

\begin{abstract}
The Wei-Norman technique allows to express the solution of a system of linear non-autonomous differential equations in terms of product of exponentials. In particular it enables to find a time-ordered product of exponentials by solving a set of nonlinear differential equations. The method has numerous theoretical and computational advantages, in particular in optimal control theory. We show that in the unitary case, i.e. when the solution of the linear system is given by a unitary evolution operator, the nonlinear system can be by an appropriate choice of ordering reduced to a hierarchy of matrix Riccati equations. Our findings have a particular significance in quantum control theory since pure quantum evolution is unitary.
\end{abstract}

\section{Introduction}

In two papers [1] and 2] written nearly fifty years ago, Wei and Norman developed a method for solving systems of linear differential equations with variable coefficients based on Lie-group techniques. As it is well known, solutions of non-autonomous linear systems of differential equations can be expressed in terms of time ordered exponentials. From this point of view 
one can treat Wei-Norman formulae as a way to calculate such exponentials. More generally they provide local coordinates on a smooth manifold on which acts a (finite-dimensional) Lie group of transformations in terms of exponential map from the Lie algebra.

Since then the Wei-Norman method has found numerous applications in control and system theory, see, e.g. [3], 4], [5], [6], [7], [8], [9], [10], as well as a basis for numerical approximation methods of integrating linear nonautonomous systems [11], [12], [13].

In recently rapidly developing theory of quantum optimal control, (see e.g. [15], [16], 17], [18, [19]) of particular importance is the case when the underlying Lie algebra is $\mathfrak{s u}(N)$, since the pure quantum evolution preserves the norm of a state vector. We show that in this case the Wei-Norman method leads to a hierarchy of matrix Riccati equations, provided that a proper ordering of generators (basis Lie algebra elements) is chosen. Our findings hinge substantially on the structure of the $\mathfrak{s u}(N)$ algebra and its complexification $\mathfrak{s l}(N, \mathbb{C})$, in particular on the properties of commutative ideals of the latter.

In the next section we present briefly the basics of the Wei-Norman method for general complex semisimple Lie group. In Section 3 we summarize properties of the $\mathfrak{s l}(N, \mathbb{C})$ relevant for our reasoning, in particular the structure of its abelian ideals. Results of this section are translated in Sections 4 and 5 to properties of the adjoint endomorphism and exponential function on $\mathfrak{s l}(N, \mathbb{C})$. The final result for the Wei-Norman formulae in the case of $\mathfrak{s l}(N, \mathbb{C})$ is given in Section 6 , The corresponding results for $\mathfrak{s u}(N)$ is easy to recover by restriction to anti-Hermitian generators. In Section 7 we give explicit formulae for low dimensions.

\section{General Wei-Norman method}

Let $G$ be a $n$-dimensional Lie group and $\mathfrak{g}$ - its Lie algebra. We assume in the following that $\mathfrak{g}$ is complex and simple. Let also $\mathbb{R} \ni t \mapsto M(t) \in \mathfrak{g}$ be a curve in $\mathfrak{g}$ and $K(t)$ - a curve in $G$ given by the differential equation

$$
\frac{d}{d t} K(t)=M(t) K(t), \quad K(0)=I .
$$

In $\mathfrak{g}$ we choose some basis $X_{k}, k=1, \ldots, n$ in which $M(t)$ takes the form

$$
M(t)=\sum_{k=1}^{n} a_{k}(t) X_{k},
$$


and look for the solution $K(t)$ in the form

$$
K(t)=\prod_{k=1}^{n} \exp \left(u_{k}(t) X_{k}\right)
$$

involving $n$ unknown functions $u_{k}$. Differentiating (3) we get

$$
\begin{aligned}
K^{\prime} & =\sum_{l=1}^{n} u_{l}^{\prime} \prod_{k=1}^{l-1} \exp \left(u_{k} X_{k}\right) X_{l} \prod_{k=l}^{n} \exp \left(u_{k} X_{k}\right) \\
& =\sum_{l=1}^{n} u_{l}^{\prime} \prod_{k=1}^{l-1} \exp \left(u_{k} X_{k}\right) X_{l} \prod_{k=l-1}^{1} \exp \left(-u_{k} X_{k}\right) \prod_{k=1}^{n} \exp \left(u_{k} X_{k}\right) \\
& =\sum_{l=1}^{n} u_{l}^{\prime} \prod_{k=1}^{l-1} \operatorname{Ad}_{\exp \left(u_{k} X_{k}\right)} \cdot X_{l} K=\sum_{l=1}^{n} u_{l}^{\prime} \prod_{k<l} \exp \left(u_{k} \operatorname{ad}_{X_{k}}\right) \cdot X_{l} K
\end{aligned}
$$

where by ' we denoted the differentiation with respect to $t$ and Ad is the adjoint action of $G$ on $\mathfrak{g}$,

$$
\operatorname{Ad}_{g} \cdot X:=g X g^{-1}, \quad g \in G, \quad X \in \mathfrak{g} .
$$

In the last equality we used used $\operatorname{Ad}_{\exp (b X)}=\exp \left(b \operatorname{ad}_{X}\right)$, where $\operatorname{ad}_{X}=[X, \cdot]$ is the adjoint action of $\mathfrak{g}$ on itself.

Comparing (1) i (41) we obtain

$$
M(t)=\sum_{l=1}^{n} u_{l}^{\prime} \prod_{k<l} \exp \left(u_{k} \operatorname{ad}_{X_{k}}\right) \cdot X_{l} .
$$

Both sides of (66) are elements of $\mathfrak{g}$ and expanding both in the basis $X_{k}$ we arrive at a system of coupled differential equations for $u_{k}$ in terms of $a_{l}$, $k, l=1, \ldots, n$.

It is worthwhile to rewrite ([6]) in more compact form. Let us denote

$$
A^{(l)}=\prod_{k<l} \exp \left(u_{k} \operatorname{ad}_{X_{k}}\right)
$$

and, consequently

$$
A^{(l)} \cdot X_{l}=\sum_{j=1}^{n} A_{j l}^{(l)} X_{j}
$$

Now (66) can be written as

$$
\sum_{j=1}^{n} a_{j} X_{j}=\sum_{l=1}^{n} u_{l}^{\prime} \sum_{j=1}^{n} A_{j l}^{(l)} X_{j}=\sum_{j=1}^{n}\left(\sum_{l=1}^{n} A_{j l}^{(l)} u_{l}^{\prime}\right) X_{j},
$$


hence

$$
a_{j}=\sum_{l=1}^{n} A_{j l}^{(l)} u_{l}^{\prime},
$$

or in a compact form

$$
\mathbf{a}=A \mathbf{u}^{\prime}
$$

where $A$ is an $n \times n$ matrix with elements $A_{j l}=A_{j l}^{l}$, i.e., its $l$-th column is equal to the $l$-th column of the matrix $A^{(l)}$, c.f. (8), and a nad $\mathbf{u}$ are the vectors of the coefficients of $M$ in (2) and the unknowns $u_{k}$. If $A$ is invertible we obtain thus a system of (nonlinear) differential equations solved for the first derivatives

$$
\mathbf{u}^{\prime}=A^{-1} \mathbf{a} .
$$

\section{$3 \quad$ The structure of $\mathfrak{s l}(N, \mathbb{C})$ algebra}

Let us start with a brief summary of the structure of simple Lie algebras [20].

Each complex simple Lie algebra $\mathfrak{g}$ can be decomposed into the root spaces with respect to a chosen Cartan subalgebra $\mathfrak{h}$ (a maximal commutative subalgebra of $\mathfrak{g}$ )

$$
\mathfrak{g}=\mathfrak{h} \oplus \bigoplus_{\alpha \in \Delta} \mathfrak{g}_{\alpha}
$$

where the one-dimensional root spaces are defined as

$$
\mathfrak{g}_{\alpha}:=\{X \in \mathfrak{g}:[H, X]=\alpha(H) X \forall H \in \mathfrak{h}\}
$$

The linear forms $\alpha \in \mathfrak{h}^{*}$ (the dual space to the algebra $\mathfrak{h}$ ) are called roots and the element $X_{\alpha}$ spanning the subspace $\mathfrak{g}_{\alpha}$ is called the root vector. By fixing a basis $\left\{H_{1}, \ldots, H_{r}\right\}$ in $\mathfrak{h}$ we can identify the set of roots $\Delta$ with a set of $r$-dimensional vectors $\alpha=\left(\alpha^{1}, \ldots \alpha^{r}\right)$, where $\alpha^{k}=\alpha\left(H_{k}\right)$. The number $r=\operatorname{dim} \mathfrak{h}$ is called the rank of $\mathfrak{g}$. Among the roots we can find a set $\Pi$ of $r$ positive simple roots, $\Pi=\left\{\alpha_{1}, \ldots \alpha_{r}\right\}$, such that for each $\alpha \in \Delta$

$$
\alpha=\sum_{i=1}^{r} m_{i} \alpha_{i},
$$

where either all $m_{i}$ are nonnegative (such roots $\alpha$ for a set of positive roots $\Delta_{+}$) or all $m_{i}$ are nonpositive (such roots $\alpha$ forming the set $\Delta_{-}$of negative roots). There is one-to-one correspondence between positive and negative roots: for each positive root $\alpha$ the is a negative one $-\alpha$. 
The root spaces have the following property

$$
\left[\mathfrak{g}_{\alpha}, \mathfrak{g}_{\beta}\right] \subset \mathfrak{g}_{\alpha+\beta},
$$

(for $\beta=-\alpha$ we have $\left[\mathfrak{g}_{\alpha}, \mathfrak{g}_{-\alpha}\right] \subset \mathfrak{h}$ ).

In terms of positive and negative roots the decomposition (13) can be rewritten as

$$
\mathfrak{g}=\mathfrak{n}_{-} \oplus \mathfrak{h} \oplus \mathfrak{n}_{+}, \quad \mathfrak{n}_{ \pm}=\bigoplus_{\alpha \in \Delta_{ \pm}} \mathfrak{g}_{\alpha}
$$

The subalgebras

$$
\mathfrak{b}_{ \pm}=\mathfrak{h} \oplus \mathfrak{n}_{ \pm}
$$

are called the Borel subalgebras relative to the Cartan subalgebra $\mathfrak{h}$.

In the following we take $G=S L(N, \mathbb{C})$ and $\mathfrak{g}=\mathfrak{s l}(N, \mathbb{C})$. In this case $n=N^{2}-1$. To construct a basis in $\mathfrak{g}$ we define

$$
S_{k l}=e_{k} e_{l}^{\dagger},
$$

where $e_{k}, k=1, \ldots, N$, is the standard basis in $\mathbb{C}^{N}$, ie., $\left(e_{k}\right)_{l}=\delta_{k l}$. The commutation relations for $S_{k l}$ read

$$
\left[S_{i j}, S_{k l}\right]=\delta_{k j} S_{i l}-\delta_{i l} S_{k j}
$$

For $k \neq l$ elements $S_{p q}$ are the root vectors. The root corresponding to roots space spanned by $S_{p q}$ will be denoted by $\alpha_{p q}$. From $S_{k l}$ we construct a basis $X_{m}$ by the following renumbering

$$
\begin{aligned}
& X_{m}=S_{p q}, \quad m=\frac{(N+q-1)(N-q)}{2}+p, \quad \text { for } p<q, \\
& X_{m}=S_{l l}-S_{l+1, l+1}, \quad m=\frac{1}{2} N(N-1)+l, \quad l=1, \ldots, N-1, \\
& X_{m}=S_{q p}, \quad m=N^{2}-\frac{(N+q-1)(N-q)}{2}+p, \quad \text { for } p<q .
\end{aligned}
$$

One may choose roots $\alpha_{p q}$ corresponding to root vectors $S_{p q}$ for $p<q$ to be the positive roots. Then the matrices (21) and (23) generate maximal nilpotent subalgebras $\mathfrak{n}_{+}$and $\mathfrak{n}_{-}$respectively (conjugated with respect to the standard Hermitian structure on $\mathbb{C}^{N}$ ), whereas (22) generate the Cartan subalgebra of $\mathfrak{s l}(N, \mathbb{C})$. In this basis $\mathfrak{n}_{+}$is the set of strictly upper triangular matrices and $\mathfrak{n}_{-}$is the set of strictly lower triangular matrices.

The set $\Pi$ of positive simple roots consist of elements of $\alpha_{l, l+1}$ for $l=$ $1, \ldots, N-1$. 
In what follows we will show many useful features of this basis. First observe that according to (21), $\mathfrak{n}_{+}:=\operatorname{span}\left\{X_{1}, \ldots, X_{N(N-1) / 2}\right\}$ and the order of root vectors spanning $\mathfrak{n}_{+}$corresponds to the following order of roots:

$$
\alpha_{i, j} \succ \alpha_{k, l} \Leftrightarrow j<l \text { or }(j=l \text { and } i>k) \text {. }
$$

Analogously $\mathfrak{n}_{-}:=\operatorname{span}\left\{X_{N(N+1) / 2}, \ldots, X_{N^{2}-1}\right\}$ and the order of root vectors spanning $\mathfrak{n}_{-}$corresponds to the following order of roots:

$$
\alpha_{i, j} \succ \alpha_{k, l} \Leftrightarrow i>k \text { or }(i=k \text { and } j<l) .
$$

Recall, that by the theorem of Lie [20], if $L$ is a nilpotent Lie algebra of dimension $r$ then there exist a sequence of ideals $I_{k}$ fulfilling

$$
0=I_{0} \subset I_{1} \subset \ldots \subset I_{r-1} \subset I_{r}=L
$$

and a basis $Y_{1}, \ldots, Y_{r}$ in $L$, such that

$$
I_{k}:=\operatorname{span}\left\{Y_{1}, \ldots, Y_{k}\right\} \text {. }
$$

The basis $\left\{X_{1}, \ldots, X_{N(N-1) / 2}\right\}$ of $\mathfrak{n}_{+}$defined in (21) has this property. It follows from (16) and the fact that $\alpha_{i, j}+\alpha_{k, l} \in \Delta$ provided $i=l$ or $j=$ $k$. In both cases $\alpha_{i, j}+\alpha_{k, l} \prec \alpha_{i, j}$, as can be easily checked. Thus $I_{k}:=$ $\operatorname{span}\left\{X_{1}, \ldots, X_{k}\right\}$ is an ideal in $\mathfrak{n}_{+}$for any $k=1, \ldots, \frac{1}{2} N(N-1)$.

Direct consequence of Lie theorem is that in this basis the matrix of endomorphism $\operatorname{ad}_{X_{k}}: \mathfrak{n}_{+} \rightarrow \mathfrak{n}_{+}$is strictly upper triangular [20]. Moreover, according to (23) matrices generating $\mathfrak{n}_{-}$are transpositions of matrices generating $\mathfrak{n}_{-}$ordered in the reverse order. This implies that matrices of endomorphisms ad $\operatorname{Xd}_{k}: \mathfrak{n}_{-} \rightarrow \mathfrak{n}_{-}$for $k=\frac{1}{2} N(N+1), \ldots, N^{2}-1$ are strictly lower triangular.

We may decompose $\mathfrak{n}_{+}$in a particular direct sum of subspaces to modify slightly the original Wei-Norman expansion (3). To this end let us consider the family $\left\{\mathfrak{a}_{k}\right\}, k=1, \ldots, N-1$ of subspaces (the numbering of the root vectors $X_{i}$ is the same as in (21)),

$$
\mathfrak{a}_{k}=\operatorname{span}\left\{X_{i_{k}}, X_{i_{k}+1} \ldots, X_{i_{k}+N-k-1}\right\}, \quad i_{k}=N(k-1)-\frac{k(k-1)}{2}+1 .
$$

In the standard matrix representation of $\mathfrak{s l}(N, \mathbb{C})$ the subspace $\mathfrak{a}_{k}$ consists of matrices with only nonvanishing entries above the diagonal in the $(N-k+1)$ th column.

We have $\operatorname{dim} \mathfrak{a}_{k}=N-k$ and $\mathfrak{n}_{+}=\bigoplus_{k=1}^{N-1} \mathfrak{a}_{k}$. Each $\mathfrak{a}_{k}$ is an abelian subalgebra of $\mathfrak{b}_{+}$and $\mathfrak{a}_{1}$ is an abelian ideal of $\mathfrak{b}_{+}$. Let us also define

$$
\mathfrak{b}_{k}=\bigoplus_{l=k}^{N-1} \mathfrak{a}_{l}
$$


Obviously $\mathfrak{a}_{k} \subset \mathfrak{b}_{k} \subset \mathfrak{b}_{k-1}$. Moreover, each $\mathfrak{b}_{k}$ is a subalgebra of $\mathfrak{n}_{+}$and $\mathfrak{a}_{k}$ is an abelian ideal of $\mathfrak{b}_{k}$, i.e.,

$$
\left[\mathfrak{b}_{k}, \mathfrak{b}_{k}\right] \subset \mathfrak{b}_{k}, \quad\left[\mathfrak{a}_{k}, \mathfrak{a}_{k}\right]=0, \quad\left[\mathfrak{a}_{k}, \mathfrak{b}_{k}\right] \subset \mathfrak{a}_{k}
$$

The subalgebras of $\mathfrak{n}_{-}$will be denoted by $\widetilde{\mathfrak{a}}_{k}$. Each $\widetilde{\mathfrak{a}}_{k}$ is the Hermitian conjugate of $\mathfrak{a}_{k}$ and is generated by the basis elements (23) in the following way:

$$
\widetilde{\mathfrak{a}}_{k}=\operatorname{span}\left\{X_{j_{k}}, X_{j_{k}+1} \ldots, X_{j_{k}+N-k-1}\right\}, \quad j_{k}=N(N-k)-\frac{k(k+1)}{2} .
$$

The subalgebras $\widetilde{\mathfrak{b}}_{k}$ are defined in analogy to (29) and together wit $\widetilde{\mathfrak{a}}_{k}$ they follow relations analogous to (30). We have the following decomposition of $\mathfrak{g}=\mathfrak{s l}(N, \mathbb{C})$ into semidirect sum of $2 N-1$ commuting subalgebras

$$
\mathfrak{g}=\mathfrak{h} \oplus \bigoplus_{i=1}^{N-1} \mathfrak{a}_{i} \oplus \bigoplus_{j=1}^{N-1} \widetilde{\mathfrak{a}}_{j}
$$

\section{Properties of the adjoint endomorphism}

Expression (6) consists of products of exponents of $\mathrm{ad}_{X_{k}}$. In this section we present some fundamental properties of the adjoint endomorphisms corresponding to root vectors in $\mathfrak{s l}(N, \mathbb{C})$ which are crucial for usefulness of the Wei-Norman method in case of $S L(N, \mathbb{C})$ group.

Lemma 4.1 Let $\alpha$ be the root and $X_{\alpha} \in \mathfrak{s l}(N, \mathbb{C})$ be the corresponding root vector. Then:

1. the image of $\left(\operatorname{ad}_{X_{\alpha}}\right)^{2}$ is equal to $\mathfrak{g}_{\alpha}$ and $\operatorname{ker}\left(\left(\operatorname{ad}_{X_{\alpha}}\right)^{2}\right)=\operatorname{span}\left\{\left\{X_{1}, \ldots, X_{N^{2}-1}\right\} \backslash\left\{X_{-\alpha}\right\}\right\}$.

2. $\left(\operatorname{ad}_{X_{\alpha}}\right)^{3}=0$.

Proof: First observe that statement 2 follows from 1 . To prove the latter recall that 20]

$$
\operatorname{ad}_{X_{\alpha}}\left(X_{\beta}\right)=\left[X_{\alpha}, X_{\beta}\right]= \begin{cases}H_{\alpha}, & \alpha+\beta=0 \\ N_{\alpha, \beta} X_{\alpha+\beta}, & \alpha+\beta \in \Delta \\ 0, & \text { otherwise }\end{cases}
$$


where $H_{\alpha} \in \mathfrak{h}$ corresponds to root $\alpha$ and $N_{\alpha, \beta}$ is some constant. We have

$$
\left(\operatorname{ad}_{X_{\alpha}}\right)^{2}\left(X_{\beta}\right)=\left[X_{\alpha},\left[X_{\alpha}, X_{\beta}\right]\right]= \begin{cases}-\alpha\left(H_{\alpha}\right) X_{\alpha}, & \alpha+\beta=0, \\ N_{\alpha, \beta} N_{\alpha, \alpha+\beta} X_{\alpha+\alpha+\beta}, & \alpha+\alpha+\beta \in \Delta, \\ 0, & \text { otherwise }\end{cases}
$$

Since the condition $\alpha+\alpha+\beta \in \Delta$ is never fulfilled for $\mathfrak{s l}(N, \mathbb{C})$, equation (34) implies that $\left(\operatorname{ad}_{X_{\alpha}}\right)^{2}$ sends any element of $\mathfrak{n}_{+} \cup \mathfrak{n}_{-}$into $\mathfrak{g}_{\alpha}$. And since for $H \in \mathfrak{h}$

$$
\left(\operatorname{ad}_{X_{\alpha}}\right)^{2}(H)=\left[X_{\alpha},\left[X_{\alpha}, H\right]\right]=-\alpha(H)\left[X_{\alpha}, X_{\alpha}\right]=0
$$

the only element of basis (21,23) on which $\left(\operatorname{ad}_{X_{\alpha}}\right)^{2}$ takes the nonzero value is $X_{-\alpha}$, what ends the proof of statement 1 .

Observe that if we have two commuting matrices of given nilpotency order $r$ then the sum of the matrices is also nilpotent of order $r$. It follows from the Jordan theorem [20] - the matrices can be expressed in Jordan form in the same basis and are block-diagonal with the same blocks of maximal size $r-1$. Since $\mathfrak{a}_{k}$ and $\widetilde{\mathfrak{a}}_{k}$ are commuting subalgebras, the lemma 4.1 yields:

Corollary 4.2 If $X \in \mathfrak{a}_{k}$ or $X \in \widetilde{\mathfrak{a}}_{k}$ then $\left(\operatorname{ad}_{X}\right)^{3}=0$.

Lemma 4.3 Let $\alpha$ be the root and $X_{\alpha} \in \mathfrak{s l}(N, \mathbb{C})$ be the corresponding root vector. In the basis defined by (21,23) the matrices of $\operatorname{ad}_{X_{\alpha}}$ for $\alpha \in \Delta_{+}$are strictly upper triangular and for $\alpha \in \Delta_{-}$are strictly lower triangular.

Proof: Let $X_{\alpha} \in \mathfrak{n}_{+}$. In the previous section we have mentioned the theorem of Lie, which has a direct consequence that the matrix of $\operatorname{ad}_{X_{\alpha}}: \mathfrak{n}_{+} \rightarrow \mathfrak{n}_{+}$is strictly upper triangular. So the sector of the matrix of endomorphism $\operatorname{ad}_{X_{\alpha}}$ : $\mathfrak{g} \rightarrow \mathfrak{g}$ corresponding do $\mathfrak{n}_{+}$is strictly upper triangular. Since $\left[\mathfrak{n}_{+}, \mathfrak{h}\right] \subset \mathfrak{n}_{+}$ the only nonzero matrix elements of $\operatorname{ad}_{X_{\alpha}}$ in the sector corresponding to $\mathfrak{h}$ lie above diagonal. Finally we consider the action $\operatorname{ad}_{X_{\alpha}}\left(X_{\beta}\right)$ for $X_{\beta} \in \mathfrak{n}_{-}$. The result is nonzero in two cases: $\alpha+\beta=0$ or $\alpha+\beta \in \Delta$. In the first case $\operatorname{ad}_{X_{\alpha}}\left(X_{\beta}\right) \in \mathfrak{h}$ and the corresponding matrix element is above diagonal. In te second case it may be easily checked directly from the definition (24-25), that $\alpha+\beta \prec \beta$. This finishes the proof for $X_{\alpha} \in \mathfrak{n}_{+}$. The proof for $X_{\beta} \in \mathfrak{n}_{-}$ is analogous.

Lemma 4.4 Let $X_{\alpha} \in \mathfrak{a}_{k} \subset \mathfrak{s l}(N, \mathbb{C})$ or $X_{\alpha} \in \widetilde{\mathfrak{a}}_{k} \subset \mathfrak{s l}(N, \mathbb{C})$, where $\alpha$ is the corresponding root. The subalgebras $\mathfrak{a}_{l}, \widetilde{\mathfrak{a}}_{l}$ for $l<k$ and the subalgebra $\mathfrak{b}_{k} \oplus \mathfrak{h} \oplus \widetilde{\mathfrak{b}}_{k}$ are invariant subspaces of $\operatorname{ad}_{X_{\alpha}}$. 
Proof: Let $X_{\alpha} \in \mathfrak{a}_{k}$. We consider three cases:

1. $X_{\beta} \in \mathfrak{a}_{l}, l<k$. In this case $X_{\alpha} \in \mathfrak{b}_{l}$ and $X_{\beta} \in \mathfrak{b}_{l}$, because $\mathfrak{a}_{k} \subset \mathfrak{b}_{k} \subset \mathfrak{b}_{l}$. Thus, $\operatorname{ad}_{X_{\alpha}}\left(X_{\beta}\right)=\left[X_{\alpha}, X_{\beta}\right] \in \mathfrak{b}_{l}$. On the other hand $X_{\beta} \in \mathfrak{a}_{l}$ and $\mathfrak{a}_{l}$ is an ideal in $\mathfrak{b}_{l}$, so $\operatorname{ad}_{X_{\alpha}}\left(X_{\beta}\right) \in \mathfrak{a}_{l}$.

2. $Y \in \mathfrak{b}_{k} \oplus \mathfrak{h} \oplus \widetilde{\mathfrak{b}}_{k}$. We have also $X_{\alpha} \in \mathfrak{b}_{k} \oplus \mathfrak{h} \oplus \widetilde{\mathfrak{b}}_{k}$ and the property $\operatorname{ad}_{X_{\alpha}}(Y) \in \mathfrak{b}_{k} \oplus \mathfrak{h} \oplus \widetilde{\mathfrak{b}}_{k}$ follows from the fact that $\mathfrak{b}_{k} \oplus \mathfrak{h} \oplus \widetilde{\mathfrak{b}}_{k}$ is subalgebra of $\mathfrak{g}$ and this follows directly from the definition of $\mathfrak{b}_{k}$ and $\widetilde{\mathfrak{b}}_{k}$ (see (29) ).

3. $X_{\beta} \in \widetilde{\mathfrak{a}}_{l}, l<k$. According to definitions (28) and (31) the roots are

$$
\begin{aligned}
\alpha=(i, N-k+1), & i<N-k+1, \\
\beta=(N-l+1, j), & N-l+1>j .
\end{aligned}
$$

We have

$$
\operatorname{ad}_{X_{\alpha}}\left(X_{\beta}\right)=\left[X_{\alpha}, X_{\beta}\right] \neq 0 \Longleftrightarrow \alpha+\beta \in \Delta \Longleftrightarrow i=j \text { or } k=l .
$$

Condition $k=l$ contradicts the assumption $k>l$. So the only possibility is $i=j$, what implies $\alpha+\beta=(N-l+1, N-k+l)$. Since $k>l$, we have $N-l+1>N-k+1$, so $\operatorname{ad}_{X_{\alpha}}\left(X_{\beta}\right)=X_{\alpha+\beta} \in \widetilde{\mathfrak{a}}_{l}$.

The same reasoning holds for $X_{\alpha} \in \widetilde{\mathfrak{a}}_{k}$.

Since for a given $k$ we have the following decomposition of $g$ :

$$
\mathfrak{g}=\mathfrak{a}_{1} \oplus \ldots \oplus \mathfrak{a}_{k-1} \oplus \underbrace{\left(\mathfrak{b}_{k} \oplus \mathfrak{h} \oplus \widetilde{\mathfrak{b}}_{k}\right)}_{\text {one block }} \oplus \widetilde{\mathfrak{a}}_{k-1} \oplus \ldots \oplus \widetilde{\mathfrak{a}}_{1}
$$

the lemmas 4.1, 4.3 and 4.4 yield

Corollary 4.5 Let $X_{\alpha} \in \mathfrak{a}_{k} \subset \mathfrak{s l}(N, \mathbb{C})\left(X_{\alpha} \in \widetilde{\mathfrak{a}}_{k} \subset \mathfrak{s l}(N, \mathbb{C})\right)$ be the root vector. In the basis (21) 23) the matrix of endomorphism $\operatorname{ad}_{X_{\alpha}}$ is nilpotent of order 3, strictly upper triangular (lower triangular) and block diagonal with respect to decomposition (39).

\section{Exponential function on commuting nilpo- tent subalgebras of $\mathfrak{s l}(N, \mathbb{C})$}

The corollary 4.5 implies the following 
Corollary 5.1 For $X \in \mathfrak{a}_{k}$ or $X \in \widetilde{\mathfrak{a}}_{k}$ the matrix of $\exp \left(\operatorname{ad}_{X}\right)$ is a quadratic polynomial in $\operatorname{ad}_{X}$. Moreover in the basis (21,23) the matrix of $\exp \left(\operatorname{ad}_{X}\right)$ is upper triangular (lower triangular for $X \in \widetilde{\mathfrak{a}}_{k}$ ) and block diagonal with respect to decomposition (39).

It is thus convenient to rewrite the equation (6) as a sum of terms corresponding to commuting subalgebras of decomposition (32). We define:

$$
U_{k}:=\prod_{i \in J_{k}} \exp \left(u_{i} \operatorname{ad}_{X_{i}}\right), \quad \widetilde{U}_{k}:=\prod_{i \in \widetilde{J}_{k}} \exp \left(u_{i} \operatorname{ad}_{X_{i}}\right),
$$

where $J_{k}=\left\{i_{k}, \ldots, i_{k}+N-k-1\right\}$ is the index range defined in (28), so for given $k$ index $i \in J_{k}$ numerates generators of subalgebra $\mathfrak{a}_{k}$, and $\widetilde{J}_{k}=\left\{j_{k}, \ldots, j_{k}+N-k-1\right\}$ is the index range defined in (31), so for given $k$ index $i \in \widetilde{J}_{k}$ numerates generators of subalgebra $\widetilde{\mathfrak{a}}_{k}$. For $l<N-k-1$ we also define:

$$
V_{k l}:=\prod_{i=i_{k}}^{i_{k}+l} \exp \left(u_{i} \operatorname{ad}_{X_{i}}\right), \quad \widetilde{V}_{k l}:=\prod_{i=j_{k}}^{j_{k}+l} \exp \left(u_{i} \operatorname{ad}_{X_{i}}\right),
$$

where for given $k$ index $i$ runs over first $l-1$ elements of $J_{k}$ or $\widetilde{J}_{k}$. We set

$$
U_{0}=\widetilde{U}_{N}=V_{k 0}=\widetilde{V}_{k 0}=\mathbb{1}
$$

Observe, that commutativity of subalgebras $\mathfrak{a}_{k}$ implies that:

$$
U_{k}=\prod_{i \in J_{k}} \exp \left(u_{i} \operatorname{ad}_{X_{i}}\right)=\exp \left(\sum_{i \in J_{k}} u_{i} \operatorname{ad}_{X_{i}}\right)=\exp \left(\operatorname{ad}_{\left(\sum_{i \in J_{k}} u_{i} X_{i}\right)}\right),
$$

so $U_{k}$ equals $\exp \left(\operatorname{ad}_{X}\right)$ for some $X \in \mathfrak{a}_{k}$ (analogously $\widetilde{U}_{k}$ equals $\exp \left(\operatorname{ad}{ }_{X}\right)$ for some $X \in \widetilde{\mathfrak{a}}_{k}$ ), so $U_{k}$ and $\widetilde{U}_{k}$ have the triangularity and block diagonality properties implied by corollary 5.1 and the same statement holds for $V_{k l}$ and $\widetilde{V}_{k l}$. From lemma 4.1 we have also the following crucial property:

Corollary 5.2 The terms quadratic in parameters $u_{i}$ appear only when $U_{k}$ or $V_{k l}$ acts on element of $\widetilde{\mathfrak{a}}_{k}$ for the same index $k$ and the terms quadratic in parameters $u_{i}$ appear only when $\widetilde{U}_{k}$ or $\widetilde{V}_{k l}$ acts on element of $\mathfrak{a}_{k}$ for the same index $k$.

Let $i_{\mathfrak{h}}=\frac{1}{2} N(N-1)+1$ be the number of the first element of Cartan subalgebra according to ordering (21)23). We define also:

$$
H:=\prod_{i=i_{\mathfrak{h}}}^{i_{\mathfrak{h}}+N-1} \exp \left(u_{i} \operatorname{ad}_{X_{i}}\right), \quad H_{l}:=\prod_{i=i_{\mathfrak{h}}}^{i_{\mathfrak{h}}+l} \exp \left(u_{i} \operatorname{ad}_{X_{i}}\right)
$$

where $l \in\{1, \ldots, N-2\}$ and $X_{i}$ generate Cartan subalgebra $\mathfrak{h}$ (see (22) ). $H$ and $H_{l}$ are diagonal matrices and $H_{0}=\mathbb{1}$. 


\section{Wei-Norman method in a properly chosen basis}

The equation (6) can be now rewritten in terms of operators defined in (40), (41) and (43) in the following simplified way:

$$
\begin{aligned}
M(t)= & \sum_{k=1}^{N-1} \prod_{l=0}^{k-1} U_{l} \cdot \sum_{i=0}^{N-k-2} V_{k i} u_{i_{k}+i}^{\prime} X_{i_{k}+i}+ \\
& +\prod_{j=1}^{N-1} U_{j} \cdot \sum_{i=0}^{N-2} H_{i} u_{i_{\mathfrak{h}}+i}^{\prime} X_{i_{\mathfrak{h}}+i}+ \\
& +\prod_{j=1}^{N-1} U_{j} \cdot H \cdot \sum_{k=1}^{N-1} \prod_{l=N}^{N-k+1} \widetilde{U}_{l} \cdot \sum_{i=0}^{N-k-2} \widetilde{V}_{k i} u_{j_{k}+i}^{\prime} X_{j_{k}+i} .
\end{aligned}
$$

On the other hand the matrix $M(t)$ by definition (2) is a linear combination of generators $X_{k}$ with given coefficients $a_{k}(t)$. The first step in order to solve the system of differential equations is to solve linear algebraic equations for $u_{k}^{\prime}$. This is done by matrix inversion (11) 12). We will show now, that the expression (44,46) allows for separation of the full set of equations into sectors corresponding to decomposition (32).

The first term in (44) corresponding to $k=1$ is an element of $\mathfrak{a}_{1}$. It follows from corollary 5.1 and the fact that it is a sum of terms of the form $\operatorname{ad}_{X} Y$ for $X \in \mathfrak{a}_{1}$ and $Y \in \mathfrak{a}_{1}$. We move this term to the left hand side of equation (44,46). The rest of the sum, remaining on the right hand side has a common factor $U_{1}$, so we multiply both sides by $U_{1}^{-1}$. After this operations right hand side does not contain terms proportional to elements of $\mathfrak{a}_{1}$, because is an composition of action of block diagonal operators with respect to decomposition (39) for $k=1$ acting on generators of $\mathfrak{g}$ starting from $\mathfrak{a}_{2}$. The left hand side reads

$$
U_{1}^{-1}\left(M(t)-\sum_{i=1}^{N-1} V_{k i-1} u_{i}^{\prime} X_{i}\right)=U_{1}^{-1}\left(\sum_{j=1}^{n} a_{j}(t) X_{j}-\sum_{i=1}^{N-1} V_{k i-1} u_{i}^{\prime} X_{i}\right)
$$

where we used the definition (21). Since there are no elements spanning $\mathfrak{a}_{1}$ on the right hand side, the first $N-1$ components of (47) have to vanish. These $N-1$ equations depend only on $u_{i}$ for $i<N$ (since $U_{1}$ depends only on them) and $u_{i}^{\prime}$ for $i<N$ (since $V_{k l}$ are upper triangular, what follows from corollary 5.1). Moreover $U_{1}$ is a quadratic function of function $u_{i}$, so we end up with matrix Riccati equation for functions $u_{i}$ for $i<N$. Once this system of 
equations is solved and its solutions are substituted into equation (44,46), the terms corresponding to generators of $\mathfrak{a}_{1}$ cancel, and we obtain reduced system of equations in smaller subspace of $\mathfrak{g}$. Next we apply the same procedure to the remaining sum on the right hand side of (44-46). It starts now from term spanned by generators of $\mathfrak{a}_{2}$. The same reasoning as for $\mathfrak{a}_{1}$ applies and after moving the term in question to the left hand side and multiplying both sides by $U_{2}$ we obtain a matrix Riccati equations for functions $u_{i}$ which multiply generators of $\mathfrak{a}_{2}$ in (3). We substitute the solutions of this system of equations and the main equation reduces again. We keep repeating the procedure described above until we obtain the solutions for all coefficient functions $u_{i}$ corresponding to all $\mathfrak{a}_{k}$. The solutions will always come from Riccati type equations. What remains from the sum (44,46) on the right hand side of (11) after inserting the solutions and multiplying both sides by product of all $U_{i}^{-1}$ is the following:

$$
\text { (l.h.s.) }=\sum_{i=0}^{N-2} H_{i} u_{i_{\mathfrak{h}}+i}^{\prime} X_{i_{\mathfrak{h}}+i}+H \cdot \sum_{k=1}^{N-1} \prod_{l=N}^{N-k+1} \widetilde{U}_{l} \cdot \sum_{i=0}^{N-k-2} \widetilde{V}_{k i} u_{j_{k}+i}^{\prime} X_{j_{k}+i} \text {. }
$$

First sum corresponds to Cartan subalgebra $\mathfrak{h}$. Since $\mathfrak{h}$ is commutative we have

$$
\exp \left(\operatorname{ad}_{X}\right) Y=Y, \quad \text { for } X, Y \in \mathfrak{h},
$$

so in (48) $H_{i} X_{i_{\mathfrak{h}}+i}=X_{i_{\mathfrak{h}}+i}$, what follows from definition (43). The second sum in (48) is a combination of generators of subalgebras $\widetilde{\mathfrak{a}}_{k}$ only, because is an action of diagonal operator $H$ and lower triangular operators $\widetilde{U}_{l}$ and $\widetilde{V}_{k i}$ on those generators. Thus the functions $u_{i}$ corresponding to generators of $\mathfrak{h}$ can be found by simple integration of solutions to previously found Riccati equations. After substitution of these solutions and multiplication of both sides of (48) by $H^{-1}$ we are left with equation where on both sides there are only terms proportional to generators $X_{j}$ spanning $\tilde{\mathfrak{a}}_{k}$, because all other terms have canceled. In order to find the solutions for remaining functions $u_{i}$ we proceed as follows. First we multiply both sides by $\widetilde{U}_{1}^{-1}$ which belongs on functions $u_{i}$ corresponding do $\widetilde{\mathfrak{a}}_{1}$ only. Derivatives $u_{i}^{\prime}$ on the right hand side appear only in the first term of the sum, because of the block diagonality of $\widetilde{V}_{k j}$ operators. Thus this terms separates form the rest and has to be equal to the action of $\widetilde{U}_{1}^{-1}$ on the left hand side. The fact that there are no generators of $\mathfrak{a}_{k}$ in the equation and corollary 5.2 imply that this set of equations will be linear in functions $u_{i}$. Once we solve it, the solutions may be substituted into equation, and terms proportional to generators of $\widetilde{\mathfrak{a}}_{1}$ will cancel. Then we multiply both sides by $\widetilde{U}_{2}$ and proceed in the same way obtaining the set of linear equations for functions $u_{i}$ corresponding to $\widetilde{\mathfrak{a}}_{2}$. We keep repeating the 
described procedure until we find linear equations for all remaining functions $u_{i}$.

The above described procedure enables the conversion of highly nonlinear differential equation (11) into hierarchy of matrix Riccati equations and linear matrix differential equations. This procedure is effective in the sense, that it provides an algorithm that may be applied directly. The authors have written a program in Maple which performs this procedure for any given $N$ and tested its successful performance up to $N=10$.

It is worth mentioning that the crucial ingredient for realizing the described algorithm in practice is the order of the generators (21,23). Once this base is used for computations and the inverse in (12) is successfully computed, the separation of the system of equations comes up automatically. For large $N$ computation of the inverse is the part of the algorithm with the largest computational complexity, which scales with $N$ as $N$ !. It follows from the fact, that the matrix $A$ in (11) which is in principal of dimension $\left(N^{2}-1\right) \times\left(N^{2}-1\right)$ has a special block upper triangular form with the largest block to invert of the size $(N-1) \times(N-1)$. The inversion has to be realized by Cramers rule which has the mentioned computational complexity.

\section{Examples}

\section{$7.1 S L(2, \mathbb{C})$}

For $N=2$ the system (12) reduces to one Riccati equation,

$$
u_{1}^{\prime}=a_{1}+2 a_{2} u_{1}-a_{3} u_{1}^{2},
$$

and two equations for the remaining unknowns,

$$
\begin{aligned}
& u_{2}^{\prime}=a_{2}-a_{3} u_{1}, \\
& u_{3}^{\prime}=a_{3} \mathrm{e}^{2 u_{2}},
\end{aligned}
$$

which give $u_{2}$ and $u_{3}$ by simple integrations, once solutions of (50) are known.

\section{$7.2 S L(3, \mathbb{C})$}

For $N=3$ we obtain from (12):

1. A system of two coupled Riccati equations

$$
\begin{aligned}
& u_{1}^{\prime}=a_{1}+\left(2 a_{5}-a_{4}\right) u_{1}+a_{6} u_{2}-a_{8} u_{1}^{2}-a_{7} u_{1} u_{2}, \\
& u_{2}^{\prime}=a_{2}+a_{3} u_{1}+\left(a_{4}+a_{5}\right) u_{2}-a_{8} u_{1} u_{2}-a_{7} u_{2}^{2},
\end{aligned}
$$


which for further reference we will rewrite in the form

$$
\mathbf{u}_{(1)}^{\prime}=\mathbf{c}_{(1)}+C_{(1)} \mathbf{u}_{(1)}+\mathbf{u}_{(1)} \mathbf{u}_{(1)}^{T} \mathbf{b}_{(1)},
$$

with

$$
\mathbf{u}_{(1)}=\left[\begin{array}{l}
u_{1} \\
u_{2}
\end{array}\right], \quad \mathbf{c}_{(1)}=\left[\begin{array}{l}
a_{1} \\
a_{2}
\end{array}\right], \quad \mathbf{b}_{(1)}=\left[\begin{array}{c}
-a_{8} \\
-a_{7}
\end{array}\right]
$$

and

$$
C_{(1)}=\left[\begin{array}{cc}
2 a_{5}-a_{4} & a_{6} \\
a_{3} & a_{4}+a_{5}
\end{array}\right]
$$

2. An equation for $u_{3}$ which reduces to a scalar Riccati equation upon substituting solutions of (58)

$$
u_{3}^{\prime}=\left(a_{3}-a_{8} u_{2}\right)+\left(2 a_{4}-a_{5}+a_{8} u_{1}-a_{7} u_{2}\right) u_{3}+\left(a_{7} u_{1}-a_{6}\right) u_{3}^{2},
$$

3. Equations for the coefficients of the Cartan algebra generators which are solved by single integrations once solutions of (53) and (58) are known,

$$
\begin{aligned}
& u_{4}^{\prime}=a_{4}-a_{6} u_{3}+a_{7}\left(u_{1} u_{3}-u_{2}\right) \\
& u_{5}^{\prime}=a_{5}-a_{8} u_{1}-a_{7} u_{2}
\end{aligned}
$$

4. Equations for the coefficients of the generators of the second ("lowertriangular") nilpotent subalgebra

$$
\begin{aligned}
& u_{6}^{\prime}=\left(a_{6}-a_{7} u_{1}\right) \mathrm{e}^{2 u_{4}-u_{5}}, \\
& u_{7}^{\prime}=\left(a_{7} u_{3}+a_{8}\right) u_{6} \mathrm{e}^{-u_{4}+2 u_{5}}+a_{7} \mathrm{e}^{u_{4}+u_{5}}, \\
& u_{8}^{\prime}=\left(a_{8}+a_{7} u_{3}\right) \mathrm{e}^{-u_{4}+2 u_{5},},
\end{aligned}
$$

which are solved by simple consecutive integrations.

\section{$7.3 \quad S L(4, \mathbb{C})$}

A similar structure emerges for $N=4$. The system of 15 equations (12) separates into:

1. A system three coupled Riccati equations,

$$
\mathbf{u}_{(1)}^{\prime}=\mathbf{c}_{(1)}+C_{(1)} \mathbf{u}_{(1)}+\mathbf{u}_{(1)} \mathbf{u}_{(1)}^{T} \mathbf{b}_{(1)},
$$


where

$$
\mathbf{u}_{(1)}=\left[\begin{array}{c}
u_{1} \\
u_{2} \\
u_{3}
\end{array}\right], \quad \mathbf{c}_{(1)}=\left[\begin{array}{c}
a_{1} \\
a_{2} \\
a_{3}
\end{array}\right], \quad \mathbf{b}_{(1)}=\left[\begin{array}{c}
-a_{15} \\
-a_{14} \\
-a_{13}
\end{array}\right],
$$

and

$$
C_{(1)}=\left[\begin{array}{ccc}
-a_{8}+2 a_{9} & a_{12} & a_{11} \\
a_{4} & -a_{7}+a_{8}+a_{9} & a_{10} \\
a_{5} & a_{6} & a_{7}+a_{9}
\end{array}\right]
$$

2. A system of two coupled Riccati equations,

$$
\mathbf{u}_{(2)}^{\prime}=\mathbf{c}_{(2)}+C_{(2)} \mathbf{u}_{(2)}+\mathbf{u}_{(2)} \mathbf{u}_{(2)}^{T} \mathbf{b}_{(2)},
$$

where

$$
\mathbf{u}_{(2)}=\left[\begin{array}{c}
u_{4} \\
u_{5}
\end{array}\right], \quad \mathbf{c}_{(2)}=\left[\begin{array}{c}
a_{4}-a_{15} u_{2} \\
a_{5}-a_{15} u_{3}
\end{array}\right], \quad \mathbf{b}_{(2)}=\left[\begin{array}{c}
-a_{12}+a_{14} u_{1} \\
-a_{11}+a_{13} u_{1}
\end{array}\right]
$$

and

$$
C_{(2)}=\left[\begin{array}{cc}
-a_{7}+2 a_{8}-a_{9}-a_{14} u_{2}+a_{15} u_{1} & a_{10}-u_{2} a_{13}-a_{15} \\
a_{6}-a_{14} u_{3} & a_{7}+a_{8}-a_{9}-a_{13} u_{3}+a_{15} u_{1}
\end{array}\right] .
$$

Observe that once a solution of (64) is known, the system (67) is closed since (68) and (69) are given in terms of some known functions.

3. A scalar Riccati equation,

$$
\begin{aligned}
u_{6}^{\prime} & =a_{6}-a_{12} u_{5}+a_{14} u_{1} u_{5}-a_{14} u_{3} \\
& +\left(2 a_{7}-a_{8}-u_{5} a_{11}+u_{4} a_{12}-u_{3} a_{13}+a_{13} u_{1} u_{5}-a_{14} u_{1} u_{4}+u_{2} a_{14}\right) u_{6} \\
& +\left(-a_{10}+u_{4} a_{11}-a_{13} u_{1} u_{4}+a_{13} u_{2}\right) u_{6}^{2},
\end{aligned}
$$

with coefficients depending on solutions of (64) and (67).

4. The remaining 9 equations for $u_{7}, \ldots, u_{15}$ which are solved by single interactions of functions constructed from the initial coefficients $a_{7}, \ldots, a_{15}$, and solutions of (64), (67) and (70).

\section{Acknowledgments}

The presented results are obtained in frames of the the Polish National Science Center project MAESTRO DEC-2011/02/A/ST1/00208 support of which is gratefully acknowledged by both authors. 


\section{References}

[1] J. Wei and E. Norman. Lie algebraic solution of linear differential equations. J. Math. Phys., 4(4):575-581, 1963.

[2] J. Wei and E. Norman. On global representations of the solutions of linear differential equations as a product of exponentials. Proc. Am. Math. Soc., 15(2):327-334, 1964.

[3] R. Brockett. Systems theory on groupmanifolds and coset spaces. SIAM J. Control, 10:265-284, 1972.

[4] R. Brockett. Lie algebras and lie groups in control theory. In R. Brockett D. Maine, editor, Geometric Methods in Systems Theory, Proceedings of the NATO Advanced Study Institute, D. Reidel, Dordrecht, 1973.

[5] T. Huillet, A. Monin, and G. Salut. Minimal realizations of the matrix transition lie groupfor bilinear control systems: explicit results. Systems Control Lett., 9:267-274, 1987.

[6] N. Leonard and P. Krishnaprasad. Averaging on lie groups, attitude control and drift. In Proceedings of the Twenty-Seventh Annual Conference on Information Sciences and Systems, 369-374, 1993.

[7] W. Chiou and S. Yau. Finite dimensional filters with nonlinear drift, brocketts problem on classification of finite dimensional estimation algebras. SIAM J. Control Optim., 32:297-310, 1994.

[8] N. Leonard and P. Krishnaprasad. Motion control of drift-free leftinvariant systems on lie groups. IEEE Trans. Automat. Control, 40:1539-1554, 1995.

[9] C.D. Charalambous and R.J. Elliott. Information states in stochastic control and filtering: A lie algebraic theoretic approach. IEEE Trans. Automat. Control, 45(4):653-674, 2000.

[10] J. Cariñena, J. Clemente-Gallardo, and Arturo Ramos. Motion on Lie groups and its applications in control theory. Rep. Math. Phys., 51:159170, 2003.

[11] B. Owren and A. Marthinsen. Integration methods based on canonical coordinates of the second kind. Numer. Math., 87:763-790, 2001.

[12] E. Celledoni and A. Iserles. Methods for the approximation of the matrix exponential in a Lie-algebraic setting. J. Numer Anal., 21:463-488, 2001. 
[13] A. Zanna and H. Z. Munthe-Kaas. Generalized polar decompositions for the approximation of the matrix exponential. SIAM J. Matrix Anal., 23:840-862, 2002.

[14] G. Dattoli, P. Di Lazzaro, and A. Torre. $S U(1,1), S U(2)$, and $S U(3)$ coherence-preserving Hamiltonians and time-ordering techniques. Phys. Rev. A, 35:1582-1589, 1987.

[15] J. F. Cariñena and A. Ramos. A new geometric approach to lie systems and physical applications. Acta Applicandae Mathematicae, 70:43-69, 2002 .

[16] J. F. Cariñena and A. Ramos. Applications of Lie systems in quantum mechanics and control theory. [in] Classical and quantum integrability: dedicated to Wtodzimierz Tulczyjew, vol. 59, p. 143. Institute of Mathematics, Polish Academy of Sciences, 2003.

[17] J. F. Cariñena, J. de Lucas, and A. Ramos. A geometric approach to time evolution operators of lie quantum systems. Int. J. Theor. Phys., 48:1379-1404, 2009.

[18] M. Kuna and J. Naudts. General solutions of quantum mechanical equations of motion with time-dependent Hamiltonians: A Lie algebraic approach. Rep. Math. Phys., 65(1):77 - 108, 2010.

[19] M. Nihtilä. Wei-Norman Technique for Control Design of Bilinear ODE Systems with Application to Quantum Control, [in] Jean Lévine and Philippe Müllhaupt, editors, Advances in the Theory of Control, Signals and Systems with Physical Modeling, volume 407 of Lecture Notes in Control and Information Sciences, pp 189-199. Springer Berlin/Heidelberg, 2011.

[20] J. E. Humphreys. Introduction to Lie Algebras and Representation Theory. Springer-Verlag 1980. 